\title{
Anti-Candida albicans IgG Antibodies in Children With Autism Spectrum Disorders
}

\author{
Heather K. Hughes and Paul Ashwood* \\ Department of Medical Microbiology and Immunology, and The Medical Investigation of Neurodevelopmental Disorders \\ (M.I.N.D.) Institute, University of California, Davis, Davis, CA, United States
}

The gut microbiota are known to have a profound influence on both mucosal and systemic immunity and are important for gastrointestinal (GI) function. In addition, new evidence shows that the microbiota significantly influence neurodevelopment and behavior. Immune dysfunction and GI distress are extremely common in individuals with autism spectrum disorders (ASD). A growing body of evidence suggests that individuals with ASD have significant aberrations in the composition of their gut microbiota, known as dysbiosis. However, these studies have focused on the bacterial components of the microbiota, leaving the fungal microbiota in ASD poorly studied. Increases in fungal species such as Candida albicans are associated with inflammatory bowel disorders, and have recently been implicated in several neurological disorders including schizophrenia.

OPEN ACCESS

Edited by:

Richard Eugene Frye Phoenix Children's Hospital,

United States

Reviewed by:

Robert Hendren,

University of California, San Francisco,

United States

David Quentin Beversdorf,

University of Missouri, United States

*Correspondence:

Paul Ashwood

pashwood@ucdavis.edu

Specialty section:

This article was submitted to

Child and Adolescent Psychiatry,

a section of the journa

Frontiers in Psychiatry

Received: 12 September 2018 Accepted: 05 November 2018

Published: 26 November 2018

Citation:

Hughes HK and Ashwood P (2018) Anti-Candida albicans IgG Antibodies in Children With Autism Spectrum

Disorders. Front. Psychiatry 9:627. doi: 10.3389/fpsyt.2018.00627
We aimed to determine if children with ASD exhibit elevations in antibodies that target $C$. albicans, indicating current or previous overgrowth of this fungal species. We measured anti-C. albicans immunoglobulin (lgG) in plasma from 80 children enrolled in the UC Davis MIND Institute CHARGE study. Measurements were acquired using a commercial ELISA kit. Plasma anti-C. albicans antibody positivity was found in $36.5 \%$ (19/52) of children with ASD. Anti-C. albicans antibodies in typically developing controls was (14.3\%; 4/28). Overall, ASD children had a higher rate of high-positive values compared to typically developed children with an unadjusted odds ratio of 3.45 (95\% confidence interval, 1.0409 to $11.4650 ; p=0.041$, two-tailed). Gl dysfunction was found in about half of the ASD children who were positive for anti-Candida lgG. This study provides evidence of a new microbial risk factor for ASD.

Keywords: autism, Candida, antibodies, immunity, behavior

\section{INTRODUCTION}

Autism spectrum disorders (ASD) are a group of heterogeneous neurodevelopmental disorders defined by deficiencies in social interactions, cognition and communication, and stereotypical and repetitive behaviors (1). Although specific genetic mutations account for $10-20 \%$ of causes for ASD, heritability risks are high and could include genes that lead to susceptibility for environmental exposures, or similar epigenetic mechanisms brought about by shared household exposures to environment (2). Environmental risk factors for having a child with ASD include gestational exposure to pollution and pesticides, and maternal infections and inflammation during pregnancy (3-5). ASD is also linked to familial autoimmunity and asthma (6-8) and many individuals with ASD have significant immune dysfunction $(9,10)$. Gastrointestinal (GI) dysfunction is also common in these individuals, with at least $50 \%$ of individuals with ASD experiencing GI issues (11). 
Gastrointestinal and immune dysfunction has been linked to aberrant composition of the microbiota, known as dysbiosis (12). Recently, researchers have identified significant bacterial dysbiosis in individuals with ASD (13-23). Bacterial dysbiosis can be caused by a variety of insults including frequent antibiotic use, which can also increase the risk of fungal overgrowth in the GI tract. Candida albicans is generally considered a passive commensal yeast of the GI and genitourinary tracts, however, it has polymorphic capabilities and under certain conditions, including altered competition within the gut, it is capable of transitioning to its pathogenic and invasive fungal form (24). The presence of Candida species during recolonization after antibiotics also contribute to dysbiosis (25) and are associated with GI disorders such as celiac disease and inflammatory bowel disorders (26-28). These competitive relationships of bacterial versus fungal microbiota are complex and still being investigated, as newer techniques develop to identify them (24).

Overgrowth of Candida species has been noted in ASD in a few studies utilizing culture-based techniques (29-31) and more recently in a study using sequencing techniques, which found Candida to be present in the stool of ASD children in nearly twice the numbers of typically developed children (21). Elevations of $\mathrm{d}$-arabinitol, a suspected metabolic byproduct of Candida species, was found in a study of 21 Italian children with ASD (32). D-arabinitol was also significantly reduced in ASD children after probiotic administration, and this correlated with improved behaviors including ability to concentrate (33). Fungal infections are an emerging area of research interest in ASD, and exposure can also be identified by looking at immunoglobulin (Ig) that target fungal antigens. They may be present in individuals with dysbiosis, as this may lead to breeches in intestinal barrier function and subsequent immune responses to commensal microbiota, including the production of IgG antibodies indicating current or previous overgrowth of this fungal species $(34,35)$. In schizophrenia, significantly elevated IgG antibodies to fungal microbiota have been seen, especially in males, however, they were also seen in bipolar females associated with lower cognitive scores (36). So far these antibodies have not been studied in individuals with ASD, therefore we aimed to determine if similar antibodies are over-represented in ASD children with and without GI dysfunction compared to their typically developing (TD) counterparts.

\section{METHODS}

\section{Study Participants}

Eighty participants ranging in age from 3 to 13 years old were enrolled in this study as part of the larger population based cohort Childhood Autism Risk from Genetics and Environment (CHARGE) study (37). ASD diagnoses were confirmed using the Autism Diagnostic Interview-Revised (ADI-R), and the Autism Diagnostic Observation Schedule (ADOS) at the time of enrollment. Social Communication Questionnaire (SCQ) was used to screen for characteristics of ASD in the typically developed children. Criteria for enrollment in the typically developed groups were scores of below 15 on the SCQ and above 70 on the Mullen Scales of Early Learning (MSEL) and
Vineland Adaptive Behavior Score (VABS). There were $n=$ 52 ASD subjects (median age 7.42 years (IQR: $5.17-9.42$ ); 8 females) and $n=28$ TD (median age 6.5 years (IQR: 5.588.33); 3 females). All subjects were administered the Aberrant Behavior Checklist (ABC) assessment. Parents also completed a CHARGE GI history (GIH) survey and GI symptom survey, based upon Rome III Diagnostic Questionnaire for the Pediatric Functional GI Disorders $(22,38)$ to identify symptoms of GI dysfunction including abdominal pain, gas/bloating, diarrhea, constipation, pain on stooling, vomiting, sensitivity to foods, difficulty swallowing, and blood in stool or vomit. Participants were excluded if they had inflammatory bowel disease (IBD) or other GI pathology, recent evidence of a GI infection and/or were taking medication that might alter GI function such as stool softeners which can alter motility or recent antibiotics/antifungals that can induce dysbiosis. In addition, participants with seizure disorder, genetic disorders, or other chronic diseases and/or infections were also excluded.

This study was approved by institutional review boards for the State of California and the University of California, Davis. Both written and informed consent was obtained from a legal guardian for all study participants prior to data collection in accordance with the UC Davis IRB protocol.

\section{Blood Collection and Enzyme-Linked Immunosorbent Assay}

Peripheral blood was collected from each subject in acidcitrate dextrose Vacutainers (BD Biosciences; San Jose, Ca). Blood was centrifuged and plasma harvested and stored at $-80^{\circ} \mathrm{C}$ until time of assay. Human anti-Candida albicans IgG was measured using enzyme-linked immunosorbent assay with a commercially available kit (Abcam, Cambridge, MA, USA) following the manufacturer's instructions. According to manufacturer's recommendations, 96 well plates were pre-coated with Candida capture antigens. Plasma samples were diluted 1:100 with diluent provide, and $100 \mu \mathrm{L}$ of diluted plasma, positive, negative, and cut-off controls were added to the plate in duplicate, leaving two blank wells per plate, and incubated in the dark for $1 \mathrm{~h}$ at $37^{\circ} \mathrm{C}$. Wells were aspirated and washed three times with provided washing solution. $100 \mu \mathrm{L}$ Candida albicans anti-IgG HRP Conjugate was added to all wells except for blank wells, and incubated for $30 \mathrm{~min}$ in the dark at room temperature, then washed again three times. One hundred microliter 3,3,5,5'-tetramethylbenzidine (TMB) solution was added to the wells and incubated for $15 \mathrm{~min}$, the reaction was stopped with reagent provided by the manufacturer. Absorbance was measured immediately on spectrophotometer at $450 \mathrm{~nm}$ with dual wavelength, using $620 \mathrm{~nm}$ as reference wavelength.

\section{Statistics}

Positivity was identified using background-adjusted absorbance of provided cut-off control. Background-adjusted sample absorbance above the positive control are denoted as highpositive. Comparison of qualitative variables between groups was assessed using Fisher's Exact Probability test with probability (P) of $<0.05$ considered significant. 


\section{RESULTS}

Plasma anti-C. albicans antibody positivity was found in 36.5\% (19/52) of children with ASD. There was a significantly higher percent positivity of plasma anti-C. albicans antibodies in children with ASD than in healthy TD controls (14.3\%; 4/28), with an unadjusted odds ratio of 3.45 (95\% confidence interval, 1.040 to $11.465 ; p=0.041$, two-tailed; Table 1). Nine of the 19 positive samples in children with ASD were considered highpositive, versus only one high-positive in the TD population (Figure 1). When examining ASD children with positivity for anti-Candida IgG, 9 of 19 (47\%) had symptoms of GI dysfunction, of the 33 negative for anti-Candida IgG 13 had GI symptoms (39\%), however no significant differences were observed based on presence or absence of GI symptoms in children with ASD. In comparison, 1 of 4 TD children positive for anti-Candida IgG had GI issues (25\%) and only 5 of 24 who were negative for anti-Candida IgG (21\%), however, these data are limited by small sample size of the TD group with GI symptoms.

\section{DISCUSSION}

Study of the microbiota-gut-brain axis has exploded over the past few decades, however, fungal microbiota research is still in the early stages. Fungal overgrowth has been implicated

TABLE 1 | The frequency of plasma anti-Candida albicans antibodies in children with ASD compared to typically developed children.

\begin{tabular}{lcc}
\hline Study group & $\begin{array}{c}\text { Anti-C albicans } \\
\text { IgG positive }\end{array}$ & $\begin{array}{c}\text { Anti-C albicans } \\
\text { IgG negative }\end{array}$ \\
\hline ASD & 19 & 33 \\
TD & $36.5 \%$ & $63.4 \%$ \\
& 4 & 24 \\
$\boldsymbol{P}$ value & $14.3 \%$ & $85.7 \%$ \\
OR (95\% Cl) & $\mathbf{0 . 0 4}$ & \\
\hline
\end{tabular}

$\mathrm{OR}$, odds ratio.

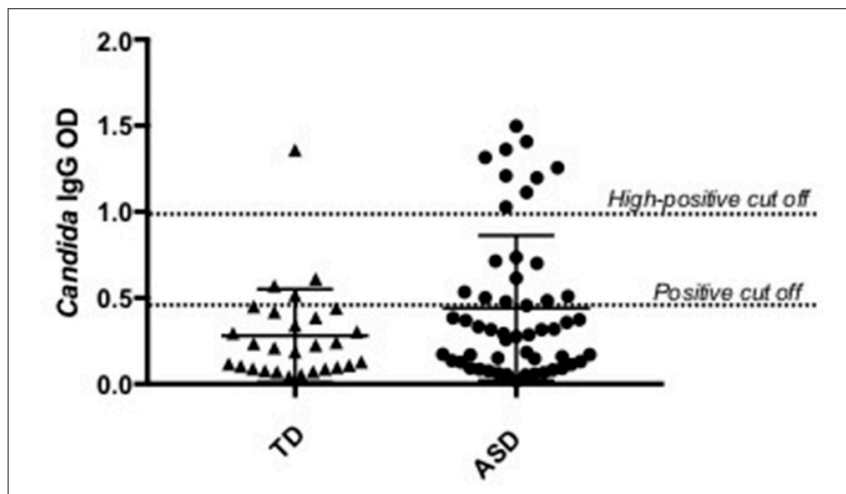

FIGURE 1 | Candida albicans plasma positivity in ASD vs. typically developing children. Nineteen children with ASD were positive for C. albicans IgG antibodies compared to four typically developing children. in inflammatory bowel disorders (39-41), schizophrenia and bipolar disorder (36), and was recently discovered within the cells of post-mortem brain tissue in Alzheimer's patients (42). Our results indicate that children with ASD have elevations in IgG against this fungal commensal, likely indicating an overgrowth of Candida albicans within the GI tract. The source of dysbiosis and Candida overgrowth in ASD is currently unknown but may be influenced by the immune dysfunction seen in many children with ASD. Alternatively, this overgrowth may be contributing to immune and GI dysfunction and the behaviors seen in ASD. This raises questions as to whether dysbiosis is present very early in life in ASD and may be involved in the etiology of ASD that need further investigation. IgG antibodies to commensal organisms become more common as individuals age, possibly due to transient intestinal breeches that might occur during enteric infections or dysbiosis from antibiotics, therefore identifying earliest exposure is a key next step to this research. Future research should attempt to identify presence of IgM antibodies which indicate current or very recent infection (35), ideally during infancy and/or early life to see if there is a relationship between infection and ASD symptom emergence.

The presence of Candida species is known to alter the assembly of microbiota after antibiotics, contributing to dysbiosis in mice (25). This suggests that if present, a different bacterial composition will occur than when absent. Therefore, if Candida is present in very early life it could interfere with initial colonization and the successions of composition during the early transitional stages of microbiota development leading to dysbiosis (43). The early microbiota are typically inherited from maternal sources, especially during vaginal delivery (43). If Candida is overgrown in mother during gestation, it could potentially be passed on to offspring as an early colonizer, interfering with normal colonization. Furthermore, the immune response to fungal overgrowth includes elevations of interleukin (IL)-17 (44). This cytokine is implicated in maternal immune activation (MIA) models of ASD, and without it ASD behaviors in MIA offspring were absent $(45,46)$. Further research utilizing animal models of fungal dysbiosis in early life or gestation could help determine the degree in which fungal microbiota may be contributing to the dysbiosis and behavioral abnormalities in offspring.

To our knowledge, this is the first study to look at antiCandida albicans IgG in children with ASD. Our recent work identified an imbalance in inflammatory versus regulatory cytokines such as transforming growth factor (TGF) beta, with alterations in the microbiota in this same population based cohort (22). Although GI symptoms were not strongly associated with Candida IgG positivity, we previously found microbiota changes in individuals with ASD irrespective of GI symptoms (22). This suggests that dysbiosis could occur even in the absence of GI symptoms. This preliminary study was initiated to guide future research on the fungal microbiota and ASD. Further validation of our results could include exploring fungal composition within the gut as well as metabolic byproducts of yeast species such as d-arabinitol and ethanol, 
and identifying associations these might have with behaviors in ASD.

\section{ETHICS STATEMENT}

This study was carried out in accordance with the recommendations of University of California Davis, IRB committee with both written informed consent from all subjects. All subjects gave written informed consent in accordance with the Declaration of Helsinki. The protocol was approved by the IRB committee at UC Davis.

\section{AUTHOR CONTRIBUTIONS}

All authors listed have made a substantial, direct and intellectual contribution to the work, and approved it for publication.

\section{REFERENCES}

1. Baio J, Wiggins L, Christensen DL, Maenner MJ, Daniels J, Warren Z, et al. Prevalence of Autism Spectrum Disorder Among Children Aged 8 Years - Autism and Developmental Disabilities Monitoring Network, 11 Sites, United States, 2014. Mmwr Surveill Summ. (2018) 67:1-23. doi: 10.15585/mmwr.ss6706al

2. Abrahams BS, Geschwind DH. Advances in autism genetics: on the threshold of a new neurobiology. Nat Rev Genet. (2008) 9:341-55. doi: 10.1038/nrg2346

3. Raz R, Roberts AL, Lyall K, Hart JE, Just AC, Laden F, et al. Autism spectrum disorder and particulate matter air pollution before, during, and after pregnancy: a nested case-control analysis within the Nurses' Health Study II Cohort. Environ Health Perspect. (2015) 123:264-70. doi: 10.1289/ehp.1408133

4. Shelton JF, Geraghty EM, Tancredi DJ, Delwiche LD, Schmidt RJ, Ritz B, et al. Neurodevelopmental disorders and prenatal residential proximity to agricultural pesticides: the CHARGE study. Environ Health Perspect. (2014) 122:1103-9. doi: 10.1289/ehp.1307044

5. Atladóttir HÓ, Henriksen TB, Schendel DE, Parner ET. Autism after infection, febrile episodes, and antibiotic use during pregnancy: an exploratory study. Pediatrics (2012) 130:e1447-54. doi: 10.1542/peds.2012-1107

6. Chen S-w, Zhong X-s, Jiang L-n, Zheng X-y, Xiong Y-q, Ma S-j, et al. Maternal autoimmune diseases and the risk of autism spectrum disorders in offspring: a systematic review and meta-analysis. Behav Brain Res. (2016) 296:61-9. doi: 10.1016/j.bbr.2015.08.035

7. Scott O, Shi D, Andriashek D, Clark B, Goez HR. Clinical clues for autoimmunity and neuroinflammation in patients with autistic regression. Dev Med Child Neurol. (2017) 59:947-51. doi: 10.1111/dmcn.13432

8. Wu S, Ding Y, Wu F, Li R, Xie G, Hou J, et al. Family history of autoimmune diseases is associated with an increased risk of autism in children: a systematic review and meta-analysis. Neurosci Biobehav Rev. (2015) 55:32232. doi: 10.1016/j.neubiorev.2015.05.004

9. Onore C, Careaga M, Ashwood P. The role of immune dysfunction in the pathophysiology of autism. Brain Behav Immun. (2012) 26:383-92. doi: 10.1016/j.bbi.2011.08.007

10. Mead J, Ashwood P. Evidence supporting an altered immune response in ASD. Immunol Lett. (2015) 163:49-55. doi: 10.1016/j.imlet.2014.11.006

11. McElhanon BO, McCracken C, Karpen S, Sharp WG. Gastrointestinal symptoms in autism spectrum disorder: a meta-analysis. Pediatrics (2014) 133:872-83. doi: 10.1542/peds.2013-3995

12. Chang C, Lin H. Dysbiosis in gastrointestinal disorders. Best Pract Res Clin Gastroenterol. (2016) 30:3-15. doi: 10.1016/j.bpg.2016.02.001

13. Parracho HM, Bingham MO, Gibson GR, McCartney AL. Differences between the gut microflora of children with autistic spectrum disorders

\section{FUNDING}

This study was funded by the NIEHS Children's Center grant (P01 ES011269), US EPA STAR program grant (R833292 and R829388), NIEHS CHARGE study (R01ES015359), NICHD (HD086669, HD090214 and U54 HD079125), National Science Foundation Graduate Research Fellowship under Grant No. 1650042, Autism Research Institute, Autism Speaks Foundation, The Boler Company Foundation, NARSAD Foundation and the Johnson Foundation.

\section{ACKNOWLEDGMENTS}

We would like to thank the staff of the CHARGE study, Destanie Rose and Emily Mills-Ko for their technical support. The commitment of the families who took part in these studies is gratefully acknowledged.

and that of healthy children. J Med Microbiol. (2005) 54(Pt 10):987-91. doi: $10.1099 / \mathrm{jmm} \cdot 0.46101-0$

14. Finegold SM, Dowd SE, Gontcharova V, Liu C, Henley KE, Wolcott RD, et al. Pyrosequencing study of fecal microflora of autistic and control children. Anaerobe (2010) 16:444-53. doi: 10.1016/j.anaerobe.2010.06.008

15. Tomova A, Husarova V, Lakatosova S, Bakos J, Vlkova B, Babinska K, et al. Gastrointestinal microbiota in children with autism in Slovakia. Physiol Behav. (2015) 138:179-87. doi: 10.1016/j.physbeh.2014.10.033

16. Williams BL, Hornig M, Buie T, Bauman ML, Cho Paik M, Wick I, et al. Impaired carbohydrate digestion and transport and mucosal dysbiosis in the intestines of children with autism and gastrointestinal disturbances. PLoS ONE (2011) 6:e24585. doi: 10.1371/journal.pone.0024585

17. Kang DW, Park JG, Ilhan ZE, Wallstrom G, Labaer J, Adams JB, et al. Reduced incidence of Prevotella and other fermenters in intestinal microflora of autistic children. PLoS ONE (2013) 8:e68322. doi: 10.1371/journal.pone.0068322

18. Williams BL, Hornig M, Parekh T, Lipkin WI. Application of novel PCR-based methods for detection, quantitation, and phylogenetic characterization of Sutterella species in intestinal biopsy samples from children with autism and gastrointestinal disturbances. MBio (2012) 3:11. doi: 10.1128/mBio.00261-11

19. Wang L, Christophersen CT, Sorich MJ, Gerber JP, Angley MT, Conlon MA. Increased abundance of Sutterella spp, and Ruminococcus torques in feces of children with autism spectrum disorder. Mol Autism. (2013) 4:42. doi: 10.1186/2040-2392-4-42

20. Wang L, Christophersen CT, Sorich MJ, Gerber JP, Angley MT, Conlon MA. Low relative abundances of the mucolytic bacterium Akkermansia muciniphila and Bifidobacterium spp. in feces of children with autism. Appl Environ Microbiol. (2011) 77:6718-21. doi: 10.1128/AEM.05212-11

21. Strati F, Cavalieri D, Albanese D, De Felice C, Donati C, Hayek J, et al. New evidences on the altered gut microbiota in autism spectrum disorders. Microbiome (2017) 5:24. doi: 10.1186/s40168-017-0242-1

22. Rose DR, Yang H, Serena G, Sturgeon C, Ma B, Careaga M, et al. Differential immune responses and microbiota profiles in children with autism spectrum disorders and co-morbid gastrointestinal symptoms. Brain Behav Immun. (2018) 70:354-68. doi: 10.1016/j.bbi.2018.03.025

23. Luna RA, Oezguen N, Balderas M, Venkatachalam A, Runge JK, Versalovic J, et al. Distinct microbiome-neuroimmune signatures correlate with functional abdominal pain in children with autism spectrum disorder. Cell Mol Gastroenterol Hepatol. (2017) 3:218-30. doi: 10.1016/j.jcmgh.2016.11.008

24. Mukherjee PK, Sendid B, Hoarau G, Colombel JF, Poulain D, Ghannoum MA. Mycobiota in gastrointestinal diseases. Nat Rev Gastroenterol Hepatol. (2015) 12:77-87. doi: 10.1038/nrgastro.2014.188

25. Erb Downward JR, Falkowski NR, Mason KL, Muraglia R, Huffnagle GB. Modulation of post-antibiotic bacterial community reassembly and host response by Candida albicans. Sci Rep. (2013) 3:2191. doi: 10.1038/srep02191 
26. Li Q, Wang C, Tang C, He Q, Li N, Li J. Dysbiosis of gut fungal microbiota is associated with mucosal inflammation in Crohn's disease. J Clin Gastroenterol. (2014) 48:513-23. doi: 10.1097/MCG.0000000000000035

27. Gerard R, Sendid B, Colombel JF, Poulain D, Jouault T. An immunological link between Candida albicans colonization and Crohn's disease. Critic Rev Microbiol. (2015) 41:135-9. doi: 10.3109/1040841X.2013.8 10587

28. Harnett J, Myers SP, Rolfe M. Significantly higher faecal counts of the yeasts candida and saccharomyces identified in people with coeliac disease. Gut Pathogens (2017) 9:26. doi: 10.1186/s13099-017-0173-1

29. Kantarcioglu AS, Kiraz N, Aydin A. Microbiota-gut-brain axis: yeast species isolated from stool samples of children with suspected or diagnosed autism spectrum disorders and in vitro susceptibility against nystatin and fluconazole. Mycopathologia (2016) 181:1-7. doi: 10.1007/s11046-015-9949-3

30. Iovene MR, Bombace F, Maresca R, Sapone A, Iardino P, Picardi $\mathrm{A}$, et al. Intestinal dysbiosis and yeast isolation in stool of subjects with autism spectrum disorders. Mycopathologia (2017) 182:349-63. doi: 10.1007/s11046-016-0068-6

31. Adams JB, Johansen LJ, Powell LD, Quig D, Rubin RA. Gastrointestinal flora and gastrointestinal status in children with autism-comparisons to typical children and correlation with autism severity. BMC Gastroenterol. (2011) 11:22. doi: 10.1186/1471-230X-11-22

32. Noto A, Fanos V, Barberini L, Grapov D, Fattuoni C, Zaffanello M, et al. The urinary metabolomics profile of an Italian autistic children population and their unaffected siblings. J Matern Fetal Neonatal Med. (2014) 27(sup2):46-52. doi: 10.3109/14767058.2014.954784

33. Kaluzna-Czaplinska J, Blaszczyk S. The level of arabinitol in autistic children after probiotic therapy. Nutrition (2012) 28:124-6. doi: 10.1016/j.nut.2011.08.002

34. Zimmermann K, Haas A, Oxenius A. Systemic antibody responses to gut microbes in health and disease. Gut Microbes (2012) 3:42-7. doi: 10.4161 /gmic. 19344

35. Janeway CJ, Travers P, Walport M. The Distribution and Functions of Immunoglobulin Isotypes. Immunobiology: The Immune System in Health and Disease. 5th edn. New York, NY: Garland Science (2001).

36. Severance EG, Gressitt KL, Stallings CR, Katsafanas E, Schweinfurth LA, Savage CL, et al. Candida albicans exposures, sex specificity and cognitive deficits in schizophrenia and bipolar disorder. NPJ schizophr. (2016) 2:16018. doi: $10.1038 /$ npjschz.2016.18

37. Hertz-Picciotto I, Croen LA, Hansen R, Jones CR, van de Water J, Pessah IN. The CHARGE study: an epidemiologic investigation of genetic and environmental factors contributing to autism. Environ Health Perspect. (2006) 114:1119-25. doi: 10.1289/ehp.8483
38. Walker LS C-DA, Rasquin-Weber A. Questionnaire on Pediatric Gastrointestinal Symptoms, Rome III Version (QPGS-RIII). Rome III: The Functional Gastrointestinal Disorders. McLean, VA: Degnon Associates, Inc (2006).

39. Standaert-Vitse A, Sendid B, Joossens M, Francois N, Vandewalle-El Khoury $\mathrm{P}$, Branche J, et al. Candida albicans colonization and ASCA in familial Crohn's disease. Am J Gastroenterol. (2009) 104:1745-53. doi: 10.1038/ajg.2009.225

40. Zwolinska-Wcislo M, Brzozowski T, Budak A, Kwiecien S, Sliwowski Z, Drozdowicz D, et al. Effect of Candida colonization on human ulcerative colitis and the healing of inflammatory changes of the colon in the experimental model of colitis ulcerosa. J Physiol Pharmacol. (2009) 60:107-18. Available online at: http://www.jpp.krakow.pl/

41. Tamboli CP, Neut C, Desreumaux P, Colombel JF. Dysbiosis in inflammatory bowel disease. Gut (2004) 53:1-4. doi: 10.1136/gut.53.1.1

42. Pisa D, Alonso R, Rabano A, Rodal I, Carrasco L. Different brain regions are infected with fungi in Alzheimer's disease. Sci Rep. (2015) 5:15015. doi: $10.1038 /$ srep 15015

43. RodrÍguez JM, Murphy K, Stanton C, Ross RP, Kober OI, Juge N, et al. The composition of the gut microbiota throughout life, with an emphasis on early life. Microb Ecol Health Dis. (2015) 26:26050. doi: 10.3402/mehd.v26. 26050

44. Conti HR, Gaffen SL. IL-17-mediated immunity to the opportunistic fungal pathogen Candida albicans. J Immunol. (2015) 195:780-8. doi: 10.4049/jimmunol.1500909

45. Choi GB, Yim YS, Wong H, Kim S, Kim H, Kim SV, et al. The maternal interleukin-17a pathway in mice promotes autism-like phenotypes in offspring. Science (2016) 351:933-9. doi: 10.1126/science.aa d0314

46. Kim S, Kim H, Yim YS, Ha S, Atarashi K, Tan TG, et al. Maternal gut bacteria promote neurodevelopmental abnormalities in mouse offspring. Nature (2017) 549:528-32. doi: 10.1038/nature2 3910

Conflict of Interest Statement: The authors declare that the research was conducted in the absence of any commercial or financial relationships that could be construed as a potential conflict of interest.

Copyright (C) 2018 Hughes and Ashwood. This is an open-access article distributed under the terms of the Creative Commons Attribution License (CC BY). The use, distribution or reproduction in other forums is permitted, provided the original author(s) and the copyright owner(s) are credited and that the original publication in this journal is cited, in accordance with accepted academic practice. No use, distribution or reproduction is permitted which does not comply with these terms. 